\title{
Is there a correlation between the aggressiveness of chronic asymptomatic prostatitis NIH category IV and the Gleason score in patients with prostate cancer?
}

\author{
Erdogan Aglamis ${ }^{1}$; Cavit Ceylan²; Mehmet Mustafa Akin ${ }^{3}$ \\ ${ }^{1}$ Health Sciences University, Elazig City Hospital, Clinics of Urology, Elazig, Turkey; ${ }^{2}$ Health Sciences \\ University, Ankara City Hospital, Clinics of Urology, Ankara, Turkey; ${ }^{3}$ Firat University Medical Faculty, \\ Department of Pathology, Elazig, Turkey
}

Cite as: Can Urol Assoc J 2020 June 5; Epub ahead of print. http://dx.doi.org/10.5489/cuaj.6284

Published online June 5, 2020

$* * *$

\begin{abstract}
Introduction: We evaluated the correlation between the International Society of Urological Pathology (ISUP) grades and the aggressiveness grades of prostate inflammation in newly diagnosed prostate cancer patients with chronic asymptomatic prostatitis National Institiutes of Health (NIH) category IV (CAPNIHIV).

Methods: The study comprised 357 consecutive patients with prostate cancer in whom a cancer diagnosis had been made via a prostate needle biopsy. Histological sections of the prostate biopsy specimens of the patients were reviewed and scored. Prostatic inflammation was scored using the aggressiveness grade of inflammation. The associations between the ISUP grades and the aggressiveness grades of inflammation were analyzed using logistic regression. The limitations of the study were its retrospective design and the limited number of cases.
\end{abstract}

Results: In 110 (31\%) patients, CAPNIHIV was detected: 56 (51\%) patients had a grade 0 aggressiveness score, 34 (31\%) patients had a grade 1 aggressiveness score, and $20(18 \%)$ patients had a grade 2 aggressiveness score. The patients who had prostatic inflammation had a 1.65 times (95\% confidence interval [CI] 1.05-2.61) greater likelihood of a high ISUP grade (grade $\geq 3$ ) compared with the patients who did not have prostatic inflammation. The association between the ISUP grade and the aggressiveness grade of inflammation was more pronounced for a grade 2 aggressiveness score ( $n=20$; odds ratio 2.97; 95\% CI 1.14-7.71). Conclusions: In prostate cancer patients with CAPNIHIV, there was a positive correlation between the inflammation aggressiveness grade and the ISUP grade. The aggressiveness of intraprostatic inflammation may be an important morphological factor affecting the Gleason score. 


\section{Introduction}

Prostate cancer is one of the most common types of cancer diagnosed by urologists, and the prostate biopsy is the standard diagnostic method for prostate cancer. ${ }^{1}$ Prostatic inflammation is one of the most frequent findings on histopathological examination of prostate biopsy specimens. ${ }^{2,3}$ According to the classification of the National Institutes of Health $(\mathrm{NIH})$, asymptomatic inflammatory prostatitis, or NIH category IV prostatitis, is described as the presence of inflammatory cells in the histopathological examination of prostate specimens or in expressed prostatic secretions. , $^{2,4}$

In some studies, it has reported that a positive relationship between chronic prostate inflammation and prostate cancer risk. ${ }^{5-8}$ In case-controlled trials, the presence of the Trichomonas vaginalis antibody was found to be positively associated with the incidence of prostate cancer. ${ }^{9}$ Similarly, it has been reported that sexually transmitted infections, such as syphilis and gonorrhea, increase the risk of prostate cancer. ${ }^{10}$ It can be deduced that these infectious agents may increase the risk of prostate cancer by leading to chronic inflammation in the prostate. ${ }^{11}$

Gleason scoring is the most common method for the histopathological evaluation of prostate cancer. ${ }^{12}$ Furthermore, the Gleason score is one of the most important prognostic factors used in predicting the prognosis and choice of treatment for prostate cancer. ${ }^{13}$ A new Gleason grading system consisting of 5 grades was defined for prostatic carcinoma at the 2014 International Society of Urological Pathology (ISUP) Consensus Conference: grade 1 (Gleason score $\leq 3+3$ ), grade 2 (Gleason score $3+4$ ), grade 3 (Gleason score $4+3$ ), grade 4 (Gleason score 3+5, 5+3,4+4), and grade 5 (Gleason score 9-10). ${ }^{14,15}$

There are no adequate studies in the literature investigating the correlation between the ISUP grades and the aggressiveness grades of inflammation. In our study, we evaluated the association between the ISUP grades and the aggressiveness grades of inflammation in newly diagnosed prostate cancer patients with asymptomatic inflammatory prostatitis.

\section{Methods}

The present study protocol was reviewed and approved by the Institutional Review Board of Turkiye Yuksek Ihtisas Training and Research Hospital (Date:12.11.2013, approval No: 311).

\section{Patients and study design}

In this retrospective study, the data of patients from whom 12 core biopsies were taken over a five-year period under the guidance of transrectal ultrasonography were evaluated. The medical history of each patient was obtained before taking the prostate biopsies, which were evaluated from clinical and laboratory perspectives in detail. The complete blood count, urine analysis, and serum total prostate-specific antigen (tPSA) level of each patient was measured. Digital rectal examination and uroflowmetry were performed, and an International Prostate Symptom Score was calculated. Under the guidance of transrectal ultrasonography, the patients with abnormal digital rectal examination findings and/or prostate cancer suspicion because of high serum tPSA levels ( $\geq 4 \mathrm{ng} / \mathrm{ml}$ ) underwent prostate biopsies. The prostate volumes of the patients were calculated using transrectal ultrasonography. 


\section{CUAJ - Original Research \\ Aglamis et al Association between ISUP and aggressiveness grades of prostate inflammation}

There were 364 patients whose histopathologic examinations showed prostate cancer. The study exclusion criteria were a history of clinical prostatitis before the prostate biopsy, a catheterization history due to urinary retention, and a rebiopsy due to an atypical small acinar proliferation or high-grade prostatic intraepithelial neoplasia. Of these 364 patients, 7 were excluded: 2 patients who had had a history of prostatitis before the prostate biopsy and 3 patients to whom catheterization had been applied due to acute urinary retention were not included in the study. In addition, 2 patients who had undergone rebiopsies because atypical small acinar proliferation had been found in their initial biopsies were not included in the study. Accordingly, a total of 357 patients were included in the study.

Our work was based on the new Gleason grading system, which had been assessed by the ISUP. ${ }^{14,15}$ The new ISUP grades of the patients included in the study were determined. In their study, Gurel et al. classified cases as low grade (Gleason sum $<7$ ) and high grade (Gleason sum 7-10), while Platz et al. classified cases as ISUP grades 1-2 and ISUP grades $3-5 .{ }^{16,5}$ Similarly, in our study, the patients with ISUP grades $1-2$ were classified as having a low ISUP grade, and the patients with ISUP grades 3-5 were classified as having a high ISUP grade.

The age, ISUP grade, tPSA value, and prostate volume of each patient were recorded. Hematoxylin-eosin-stained histological sections of the prostate biopsy specimens of the 357 patients were reviewed and scored by two experienced pathologists. The pathologists performing the evaluation were not informed about the purpose of the study. Prostatic inflammation was scored for the aggressiveness grade of inflammation using the grading system reported by Irani et al., namely, grade $0=$ no contact between glandular epithelium and inflammatory cells infiltrate (intact epithelial cells), grade $1=$ contact between glandular epithelium and inflammatory cells infiltrate (without glandular epithelium disruption), grade 2 $=$ interstitial inflammatory cells infiltrate associated with a clear, less than $25 \%$ glandular epithelium disruption in the examined material, grade $3=$ more than $25 \%$ glandular epithelium disruption in the examined material (Figure 1). ${ }^{2,17}$

Among the patients, 110 had prostate cancer together with asymptomatic inflammatory prostatitis, and they were classified as grade 0 , grade 1 , and grade 2 according to their aggressiveness scores of inflammation.

The mean age, prostate volume, and tPSA values of the patients with and without prostatic inflammation were compared. The association between the ISUP grade and the aggressiveness grade of inflammation was evaluated.

\section{Statistical analysis}

A statistical analysis was performed using SPSS $®$ software. Comparisons between the ages, prostatic volumes, and serum total PSA levels of the patients were performed by $t$-tests and one-way ANOVA tests (value of significance: $p<0.05$ ). The correlation between the tPSA and the aggressiveness grade of inflammation was determined by Pearson correlation analysis (value of significance: $\mathrm{p}<0.05$ ). The odds ratios (ORs) and 95\% confidence intervals (CIs) for the associations between the ISUP grades and the aggressiveness grades of prostatic inflammation were calculated using logistic regression analysis. 


\section{Results}

\section{Patient characteristics}

The characteristics of the 357 patients who were included in the study are presented in Table 1. In the histopathological examination, 247 (69\%) patients with prostate cancer had no asymptomatic inflammatory prostatitis, while $110(31 \%)$ patients with prostate cancer had asymptomatic inflammatory prostatitis. The aggressiveness grade distribution of the 110 patients with chronic prostatic inflammation was as follows: $56(51 \%)$ patients were grade 0 , 34 (31\%) patients were grade 1, and $20(18 \%)$ patients were grade 2 . There were no patients with an aggressiveness score of grade 3 . In one patient, there was no clear distinction between the aggressiveness scores of grades 2 and 3, so this patient was included in the patient group with aggressiveness scores of grade 2 .

There was no statistically significant difference in terms of age and prostate volume between the patients with and without asymptomatic inflammatory prostatitis. However, the tPSA values were significantly higher in the patients with prostate inflammation. When the group of patients without asymptomatic inflammatory prostatitis and the group of patients with inflammation aggressiveness scores of grades 0 and 1 were compared, no significant difference was observed between the tPSA values. In contrast, the tPSA values of the patient group with grade 2 inflammation aggressiveness were found to be significantly higher than those of all the other groups. There was no significant difference between mean tPSA values of aggressiveness grade 0 and aggressiveness grade 1 patients (Table 1). There was a positive correlation between tPSA and the aggressiveness grade of inflammation $(r=0.234, p \leq 0.001$, Pearson correlation).

Table 2 presents the distribution of patients according to ISUP grade. Of the 357 patients, 151 (42\%) patients had high ISUP grades and $206(58 \%)$ patients had low ISUP grades. Of the 247 patients without asymptomatic inflammatory prostatitis, 95 (38\%) patients had high ISUP grades and 152 (62\%) patients had low ISUP grades. Of the 110 patients with asymptomatic inflammatory prostatitis, $56(51 \%)$ patients had high ISUP grades and 54 (49\%) patients had low ISUP grades (Table 2).

\section{Association of aggressiveness of inflammation with ISUP grade}

Table 3 provides the ORs of the high ISUP grade in relation to the aggressiveness grade of inflammation. Patients with prostatic inflammation had nearly a $65 \%$ higher likelihood of a high ISUP grade (OR, 1.65; 95\% CI, 1.05-2.61) than the patients who did not have prostatic inflammation. In the patients with a grade 2 aggressiveness score, this association was significantly more prominent (OR, 2.97; 95\% CI, 1.14-7.71). When compared to the patients without prostatic inflammation, the likelihood of a high ISUP grade increased from a grade 0 aggressiveness score (OR, 1.29; 95\% CI, $0.71-2.31)$ to a grade 2 aggressiveness score (OR, 2.97; 95\% CI, 1.14-7.71; $\mathrm{p}=0.02)$ in the patients with prostatic inflammation. As the aggressivity of inflammation increased, the probability of a high ISUP grade also increased (Table 3, Graphic 1). 


\section{Discussion}

The results of our study showed that the probability of a high ISUP grade was higher in prostate cancer patients with asymptomatic inflammatory prostatitis diagnosed via a prostate needle biopsy. As the aggressiveness grade of inflammation increased, the likelihood of a high ISUP grade also increased. This relationship was considerably more prominent in the patients with a grade 2 aggressiveness score. These results support the hypothesis that asymptomatic inflammatory prostatitis affects the development of prostate cancer with a high ISUP grade.

As in many cancer types, the course of prostate cancer is different for each patient. In patients with prostate cancer, various prognostic factors and nomograms are used to predict the course that the disease will follow. The Gleason grading system used in prostate cancer is one of the most important prognostic factors and reportedly a safe indicator of survival. ${ }^{12,13}$

Prostatic inflammation is one of the most common findings in the histopathological examination of prostate biopsy specimens. ${ }^{2,3}$ In prostate biopsies, asymptomatic inflammatory prostatitis has been reported to have a ratio of $32-44 \% .{ }^{1,18,19}$ In our study, asymptomatic inflammatory prostatitis was detected in $31 \%$ of 357 patients. This rate was compatible with the rates reported in the literature.

Symptomatic prostatitis significantly increases serum tPSA levels. ${ }^{20}$ Notwithstanding, in some studies, asymptomatic inflammatory prostatitis was found not to significantly affect serum tPSA levels. ${ }^{21}$ However, the common view is that asymptomatic inflammatory prostatitis may significantly increase serum tPSA levels. ${ }^{2,4,22}$ Serum tPSA levels have also been found to be higher in patients with excess intraprostatic inflammation. ${ }^{4,23}$ Yaman et al. reported that there is a significant correlation between the aggressiveness grade of subclinic prostatic inflammation and PSA. ${ }^{23}$ In the present study, there was also a positive correlation between the aggressiveness of chronic inflammation and PSA: the serum TPSA levels were found to be significantly higher in the patients with prostate cancer whose aggressiveness grade was 2 .

Some studies have reported that there is no positive relationship between chronic inflammation and prostate cancer. ${ }^{24,25}$ However, many studies have reported a positive correlation between chronic prostate inflammation and prostate cancer risk. ${ }^{5-8,11,16}$ In a study on the impact of chronic inflammation on prostate cancer progression, it was found that chronic inflammation can increase the possibility of prostate cancer. In the study, Gurel et al. found that intraprostatic inflammation is associated with a high-grade prostate cancer etiology. ${ }^{16}$ The ISUP grade was found to be high in the patients with an elevated chronic inflammation aggressivity grade in our study, and this result supports their hypothesis. The results reported in the literature and in our work are different from those of the REDUCE trial. In the REDUCE trial, the patients with inflammation but no prostate cancer as determined by a needle biopsy had a lower likelihood of developing prostate cancer in the future. ${ }^{26}$ However, the PSA concentrations of the men included in the study ranged from $2.5 \mathrm{ng} / \mathrm{mL}$ to $10 \mathrm{ng} / \mathrm{mL}$. Furthermore, all prostate cancer patients were included in our study, regardless of their serum tPSA levels. 
In a study of Irani et al., both inflammatory infiltration and aggressiveness of prostatic inflammation were graded for asymptomatic inflammatory prostatitis. Inflammatory infiltration grading has 4 scores, grade 0 : no inflammatory cells; grade 1: inflammatory cells scattered into the stroma without lymphoid nodules. grade 2: Separate (non-associated) lymphoid nodules, grade 3: combination of infiltrate forming large areas of inflammation. ${ }^{17}$ As we aimed to investigate the correlation between the Gleason score and aggressiveness of asymptomatic inflammatory prostatitis in this study; only the aggressiveness score of prostatic inflammation was checked. But not performing the grading of inflammatory infiltration can be counted as one of the limitations of our study.

In patients with asymptomatic inflammatory prostatitis, the incidence of grade 3 aggressiveness score has been reported to be low. In a study of Irani et al., in which grading the aggressiveness of prostatic inflammation was performed, only $2(1.8 \%)$ of 111 patients with asymptomatic inflammatory prostatitis were reported to have grade 3 aggressiveness score. ${ }^{17}$ In our study, 110 patients had asymptomatic inflammatory prostatitis, but histopathological examination by experienced pathologists did not report a case of grade 3 aggressiveness score. This may be due to the low incidence of grade 3 aggressiveness score.

In some studies, intraprostatic inflammation has been found to be a prognostic factor for prostate cancer. A study by Irani et al. determined that the biochemical recurrence rate was significantly high in patients with high-grade inflammation after radical prostatectomy. In their study, Irani et al. hypothesized that chronic inflammation is an independent prognostic factor for disease-free survival in patients who underwent radical prostatectomy due to localized prostate cancer. ${ }^{27}$ Meanwhile, Davidsson et al. investigated the prognostic significance of morphological changes in prostate tissue during prostate cancer and showed that moderate or severe chronic inflammation may have prognostic significance for prostate cancer. ${ }^{28}$ The significant association between high aggressiveness-scored inflammation and high ISUP grade in our study supports these hypotheses.

The effect of the existence of asymptomatic inflammatory prostatitis on the course of prostate cancer has not been confirmed, and further research in this regard is required. The present study found support for the relationship between prostatic inflammation and prostate cancer. The limitations of the study were that the study was conducted retrospectively, the number of patients was small, and the results were not compared with the Gleason score in radical prostatectomy specimens. Another limitation of our study is the lack of infiltration grading of inflammation. The results of the study need to be prospective and should be verified using a larger patient sample.

\section{Conclusions}

This study determined that, in newly diagnosed prostate cancer patients with asymptomatic inflammatory prostatitis, the likelihood of a high ISUP grade increased as the grade of aggressiveness of inflammation increased. The aggressiveness of intraprostatic inflammation may be an important morphological factor affecting Gleason scores. Whether or not intraprostatic chronic inflammation is a prognostic criterion in prostate cancer should be considered as a future research topic. 

Association between ISUP and aggressiveness grades of prostate inflammation

\section{References}

1. Aglamis E, Tasdemir C, Ceylan C. The role of National Institutes of Health category IV prostatitis in accurately staging the newly diagnosed prostate cancer. Ir J Med Sci 2013(3); 182: 463-67.

2. Ozden C, Ozdal OL, Guzel O, et al. The correlation between serum prostate specific antigen levels and asymptomatic inflammatory prostatitis. Int Urol Nephrol 2007; 39:859-63.

3. Stimac G, Reljic A, Spajic B, et al. Aggressiveness of Inflammation in Histological Prostatitis - Correlation with Total and Free Prostate Specific Antigen Levels in Men with Biochemical Criteria for Prostate Biopsy. Scott Med J 2009; 54 (3): 8-12.

4. Stancik I, Luftenegger W, Klimpfinger M, et al. Effect of NIH-IV Prostatitis on Free and Free-to-total PSA. Eur Urol 2004; 46: 760-64.

5. Platz EA, Kulac I, Barber JR, et al. A Prospective Study of Chronic Inflammation in Benign Prostate Tissue and Risk of Prostate Cancer: Linked PCPT and SELECT Cohorts. Cancer Epidemiol Biomarkers Prev 2017; 26(10): 1549-57.

6. Fujita K, Hayashi T, Matsushita M, et al. Obesity, Inflammation, and Prostate Cancer. J Clin Med 2019; 8(2): 201

7. Hayashi T, Fujita K, Matsushita M et al. Main Inflammatory Cells and Potentials of Anti-Inflammatory Agents in Prostate Cancer. Cancers 2019; 11(8): 1153-68.

8. Puhr M, Marzo DM, Isaacs W, et al. Inflammation, Microbiota, and Prostate Cancer. Eur Urol Focus 2016;2(4): 374-82.

9. Stark JR, Judson G, Alderete JF, et al. Prospective study of Trichomonas vaginalis infection and prostate cancer incidence and mortality: Physicians'HealthStudy. $J$ Natl Cancer Inst 2009; 101: 1406-11.

10. Hayes RB, Pottern LM, Strickler H, et al. Sexual behaviour, STDs and risks for prostate cancer. Br J Cancer 2000; 82: 718-25.

11. De Nunzio C, Kramer G, Marberger M, et al. The Controversial Relationship Between Benign Prostatic Hyperplasia and Prostate Cancer: The Role of Inflammation. Eur Urol 2011; 60(1): 106-17.

12. Groll R, Fleshner NE, Sugar L, et al. Clinical significance of biopsy-derived primary Gleason score among radical prostatectomy candidates with Gleason 7 tumors. Urology 2002; 59(4): 551- 54.

13. Malavaud B, Villers A, Ravery V, et al. Role of preoperative positive apical biopsies in the prediction of specimen-confined prostate cancer after radikal retropubic prostatectomy: A multi institutional study. Eur Urol 2000; 37: 281-88.

14. Epstein JI, Egevad L, Amin MB, et al. The 2014 International Society of Urological Pathology (ISUP) Consensus Conference on Gleason Grading of Prostatic Carcinoma Definition of Grading Patterns and Proposal for a New Grading System. Am J Surg Pathol 2016; 40: 244-52.

15. Samaratunga H, Delahunt B, Yaxley J, et al. From Gleason to International Society of Urological Pathology (ISUP) grading of prostate cancer. Scand J Urol 2016; 50(5): $325-29$.

16. Gurel B, M. Lucia MS, Thompson IM, et al. Chronic Inflammation in Benign Prostate Tissue Is Associated with High-Grade Prostate Cancer in the Placebo Arm of the Prostate Cancer Prevention Trial. Cancer Epidemiol Biomarkers Prev 2014; 23(5): 847-56. 

Association between ISUP and aggressiveness grades of prostate inflammation

17. Irani J, Levillain P, Goujon JM, et al. Inflammation in benign prostatic hyperplasia: Correlation with prostate specific antigen value. J Urol 1997;157:1301-03.

18. Blumenfeld W, Tucci S, Narayan P. Incidental lymphocytic prostatitis: Selective involvement with nonmalignant glands. Am J Surg Pathol 1992; 16: 975-81.

19. Carver BS, Bozeman CB, Williams BJ, et al. The prevalance of men with national institutes of health category IV prostatitis and association with serum prostate specific antigen. J Urol 2003; 169: 589-91.

20. Battikhi MN, Ismail H, Battikhi Q. Effects of chronic bacterial prostatitis on prostate specific antigen levels total and free in patients with benign prostatic hyperplasia and prostate cancer. Int Urol Nephrol 2006; 38: 21-26.

21. Nickel JC, Downey J, Young I, et al. Asymptomatic inflammation and/or infection in benign prostatic hyperplasia. BJU Int 1999; 84 (9): 976-81.

22. Liotta RF, Tarantino ML, Melloni D. Chronic prostatitis and PSA values. Urologia 2008; 75(1):102-04.

23. Yaman O, Gogus $\mathrm{C}$, Tulunay $\mathrm{O}$, et al. Increased prostate specific antigen in subclinical prostatitis: the role of aggressiveness and extension of inflammation. Urol Int 2003;71:160-64.

24. Karakiewicz PI, Benayoun S, Begin LR, et al. The international journal of Clinical Practice 2007: Chronic inflammationis negatively associated with prostate cancer and high-grade prostatic intraepithelialneoplasia on needle biopsy. Int J Clin Pract 2007; 61(3):425-30.

25. Langston ME, Horn M, Khan S, et al. A systematic review and meta-analysis of associations between clinical prostatitis and prostate cancer: new estimates accounting for detection biast. Cancer Epidemiol Biomarkers Prev 2019; 20(10): 1594-603.

26. Moreira DM, Nickel JC, Gerber L, et al. Baseline prostate inflammation is associated with a reduced risk of prostate cancer in men undergoing repeat prostate biopsy: results from the REDUCE study. Cancer 2014; 15;120(2):190-96.

27. Irani J, Goujon JM, Ragni E, et al. High-Grade Inflammation In Prostate Cancer As A Prognostic Factor For Biochemical Recurrence After Radical Prostatectomy. Pathologist Multi Center study Group. Urology 1999; 54(3): 467-72.

28. Davidsson S, Fiorentino M, Andren O, et al. Inflammation, Focal Atrophic Lesions, and Prostatic Intraepithelial Neoplasia with Respect to Risk of Lethal Prostate Cancer. Cancer Epidemiol Biomarkers Prev 2011;20(10):2280-87. 


\section{Figures and Tables}

Fig. 1. Scoring for aggressiveness grade of chronic inflammation in prostate needle biopsy. Hematoxylin-Eosin, x200. (A) Grade $0=$ There isn't contact between glandular epithelium and inflammatory cells infiltrate ( intact epithelial cells). (B) Grade 1=There is contact between glandular epithelium and inflammatory cells infiltrate (without glandular epithelium disruption). (C) Grade 2=Interstitial inflammatory cells infiltrate associated with a clear, less than $25 \%$ glandular epithelium disruption in the examined material.

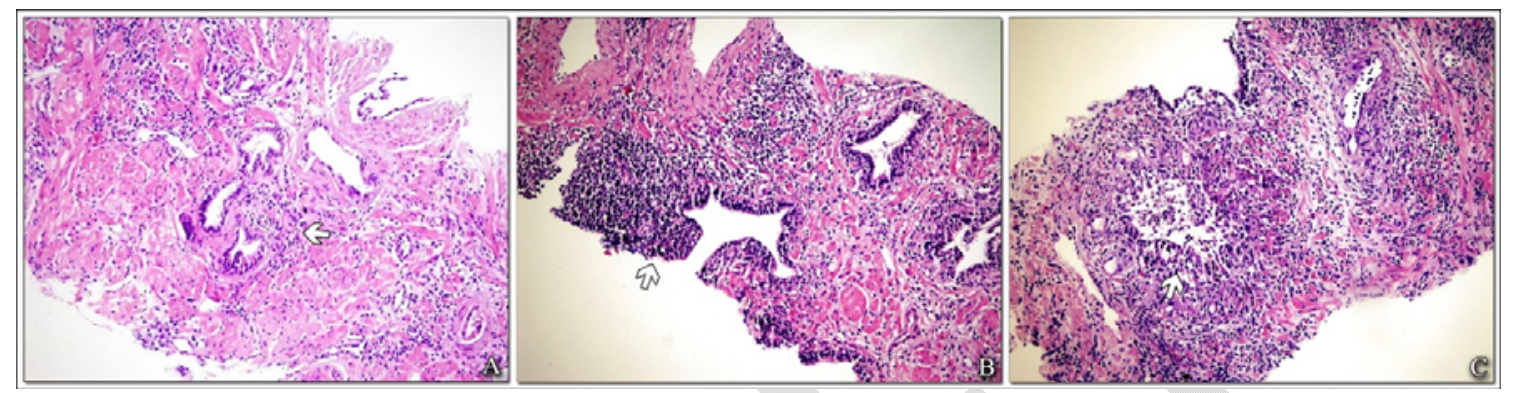

Fig 2. Associations between the aggressiveness grade of inflammation and the high International Society of Urological Pathology (ISUP) grade (ISUP grade 3-5). As the aggressivity of inflammation increased, the odds of the high ISUP grade (ISUP grade 3-5) was also increased. *Based on literature studies, cases were classified as low ISUP grade and high ISUP grade. ${ }^{5,16} \mathrm{CI}$ : confidence interval; OR: odds ratio.

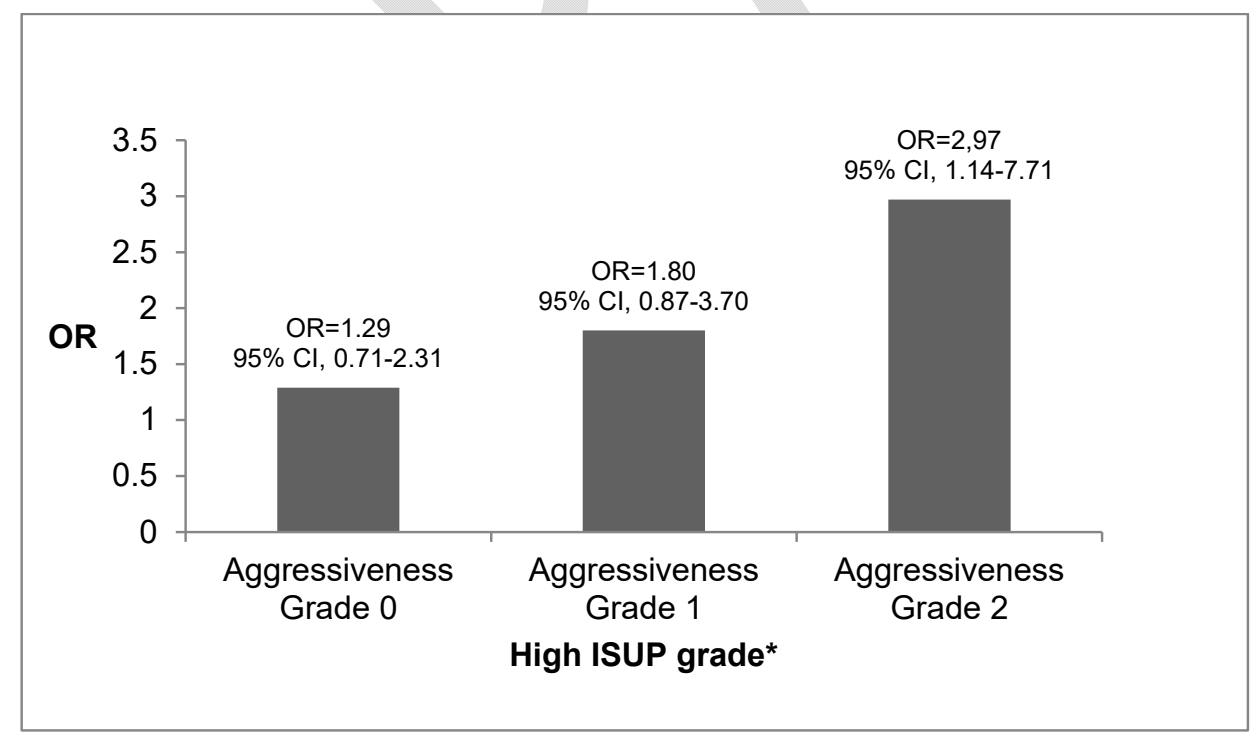




\begin{tabular}{|c|c|c|c|c|c|c|}
\hline & Total & PCa & \multicolumn{4}{|c|}{ PCa + chronic inflammation } \\
\hline & & & \multirow[t]{2}{*}{ Total } & \multicolumn{3}{|c|}{ Aggressiveness of inflammation } \\
\hline & & & & Grade 0 & Grade 1 & Grade 2 \\
\hline $\mathrm{N}(\%)$ & 357 & $247(69)$ & $110(31)$ & $56(51)$ & $34(31)$ & $20(18)$ \\
\hline Mean age & 67.7 & 67.3 & 68.6 & 68.7 & 68.8 & 67.9 \\
\hline Mean prostate volume $(\mathrm{ml})$ & 56.7 & 55.8 & 58.9 & 57.2 & 58.0 & 65.3 \\
\hline Mean PSA(ng/mL) & 9.0 & $8.2^{\mathrm{b}}$ & $10.8^{\mathrm{a}}$ & $8.7^{c}$ & $10.6^{\mathrm{d}}$ & 16.7 \\
\hline & & & & & & \\
\hline & & & & & & \\
\hline
\end{tabular}

${ }^{a} \mathrm{p}=0.003$ ( $\mathrm{p}$ value from a t-test), compared with PCa group. ${ }^{\mathrm{b}, \mathrm{c}, \mathrm{d}} \mathrm{p}<0.0001, \mathrm{p}<0.0001, \mathrm{p}<0.05$, respectively ( $\mathrm{p}$ value from a one-way Anova test), compared with aggressiveness grade 2 group. PCa: prostate cancer without asymptomatic inflammatory prostatitis; $\mathrm{PCa}+$ chronic inflammation: prostate cancer together with asymptomatic inflammatory prostatitis; PSA: prostate-specific antigen.

\begin{tabular}{|l|l|c|c|c|}
\hline \multicolumn{2}{|l|}{ Table 2. Distribution of patients according to ISUP grade } \\
\hline & & Total & PCa & PCa + chronic inflammation \\
\hline N (\%) & 357 & $247(69 \%)$ & $110(31 \%)$ \\
\hline ISUP grade & $\begin{array}{l}\text { High (ISUP 3-5), } \\
\mathrm{n}(\%)\end{array}$ & $151(42 \%)$ & $95(38 \%)$ & $56(51 \%)$ \\
\cline { 2 - 5 } & $\begin{array}{l}\text { Low (ISUP 1-2), } \\
\mathrm{n}(\%)\end{array}$ & $206(58 \%)$ & $152(62 \%)$ & $54(49 \%)$ \\
\hline
\end{tabular}

ISUP: International Society of Urological Pathology; PCa:prostate cancer without asymptomatic inflammatory prostatitis; $\mathrm{PCa}+$ chronic inflammation: prostate cancer together with asymptomatic inflammatory prostatitis. 


\begin{tabular}{|c|c|c|c|c|}
\hline & & $\mathbf{N}$ & \multicolumn{2}{|c|}{$\begin{array}{l}\text { High ISUP grade } \\
\text { (ISUP 3-5) }\end{array}$} \\
\hline & & & OR & $95 \% \mathrm{CI}$ \\
\hline Prostatic inflammation, No & & 247 & 1.00 & Reference \\
\hline Prostatic inflammation, $\mathrm{Yes}^{\mathrm{a}, \mathrm{b}}$ & & 110 & 1.65 & $1.05-2.61$ \\
\hline \multirow{3}{*}{$\begin{array}{l}\text { Aggressiveness grade of } \\
\text { inflammation }\end{array}$} & Grade 0 & 56 & 1.29 & $0.71-2.31$ \\
\hline & Grade 1 & 34 & 1.80 & $0.87-3.70$ \\
\hline & Grade $2^{b}$ & 20 & 2.97 & $1.14-7.71$ \\
\hline
\end{tabular}

${ }^{\mathrm{a}}$ All patients with any Aggressiveness grade of inflammation. ${ }^{\mathrm{b}} \mathrm{p}=0.02$. CI: confidence interval; ISUP: International Society of Urological Pathology; OR: odds ratio. 